\title{
Intrinsic breast cancer subtypes defined by estrogen receptor signalling-prognostic relevance of progesterone receptor loss
}

\author{
Lisa Braun ${ }^{1}$, Friederike Mietzsch ${ }^{1}$, Petra Seibold ${ }^{2}$, Andreas Schneeweiss ${ }^{3}$, \\ Peter Schirmacher ${ }^{1}$, Jenny Chang-Claude ${ }^{2}$, Hans Peter Sinn ${ }^{1}$ and Sebastian Aulmann ${ }^{1}$ \\ ${ }^{1}$ Institute of Pathology, Heidelberg University, Heidelberg, Germany; ${ }^{2}$ Division of Cancer Epidemiology, \\ Unit of Genetic Epidemiology, German Cancer Research Center (DKFZ), Heidelberg, Germany and \\ ${ }^{3}$ National Center for Tumour Diseases, Heidelberg University Hospital, Heidelberg, Germany
}

\begin{abstract}
The majority of luminal type breast carcinomas are slowly growing tumors with an overall favorable prognosis. However, a proportion of cases (luminal B tumors) are characterized by coactivation of growth factor receptors or non-canonical ER signaling and a poorer clinical outcome. The aim of our study was to evaluate whether the expression of proteins that are part of the ER signaling network may be used to distinguish low-risk from highrisk luminal tumors. Unsupervised hierarchical clustering of a set of proteins either involved in estrogen receptor signaling or associated with resistance to endocrine therapy was performed in a series of $\mathbf{4 4 3}$ postmenopausal breast carcinomas. Using this approach, we were able to reproduce the established classification with two distinct groups of luminal (estrogen receptor positive) tumors, one group of HER2associated tumors and a group of triple-negative tumors. However, neither proliferation nor the expression of one or more of the ER-co-factors or resistance-associated factors, but PR-expression was identified as the most important stratifier distinguishing between the two luminal groups. In fact, not only the four identified clusters were shown to be significantly associated with patient outcome, PR-expression alone or in combination with Ki-67-stains stratified ER-positive tumors into a low-risk and a high-risk group. Our data indicate that defining luminal B tumors by the presence of high-risk criteria (loss of PR-expression or increased proliferation) provides a robust and highly significant stratification of ER-positive breast carcinomas into luminal $A$ and $B$. Modern Pathology (2013) 26, 1161-1171; doi:10.1038/modpathol.2013.60; published online 5 April 2013
\end{abstract}

Keywords: breast cancer; intrinsic subtypes; ER signaling; PR; immunohistochemistry; IHC4-score

Hormonal influences are of substantial relevance not only for normal mammary gland development but also for its plasticity under different physiological situations. However, estrogen- and progesterone receptors are also frequently observed in breast carcinomas and there is substantial evidence for a key role of both steroid hormones in tumor initiation and growth. In fact, anti-estrogenic treatment either by estrogen-deprivation or selective estrogen receptor modulators still represents one of the most successful targeted therapies in cancer treatment. ${ }^{1}$ Over the past years, insight into estrogen receptor functions, its cofactors and transcriptional targets

Correspondence: Dr S Aulmann, MD, Institute of Pathology, Heidelberg University, Im Neuenheimer Feld 224, Heidelberg 69120, Germany.

E-mail: sebastian.aulmann@med.uni-heidelberg.de

Received 20 December 2012; revised 31 January 2013; accepted 2 February 2013; published online 5 April 2013 has substantially grown and suggest an intricate network of pathways influenced by estrogen receptor or growth factor signaling, regulating different cellular responses such as growth, differentiation, apoptosis, or proliferation. In recent years, a number of interacting pathways such as MAPK or PI3K/AKT signaling have been identified that may be activated in cancer cells leading to sustained growth under anti-hormonal treatment. ${ }^{2}$

Genomic profiling in combination with hierarchical clustering has led to the identification of at least four distinct molecular breast cancer subtypes. ${ }^{3,4}$ In addition to tumors containing amplifications of the ERBB2 (or HER2) gene (which have been appropriately termed ERBB2-tumors) and basal-like tumors which contain a high number of so-called triple-negative (ie, estrogen receptor, progesterone receptor, and HER2-negative by immunohistochemistry), two large groups of breast tumors (luminal A and B) were identified that were shown 
to contain the majority of hormone-receptor positive tumors but that differed in respect to proliferation, metastatic potential, and prognosis. ${ }^{5}$ Unfortunately, no reliable molecular predictors or classifiers could be extracted from any of the profiling studies that allowed a prospective designation of a given tumor sample either to the luminal A or the luminal B cluster. Although the overlap of the different genetic signatures between different studies has been disappointingly low and individual tumor samples could not be reproducibly placed in one of the different categories by applying the published signatures, ${ }^{6}$ the great merit of these molecular studies was the constant demonstration of the existence of these different molecular phenotypes, for which markers or signatures for proper designation of individual tumor samples in a prospective way are needed.

Today, immunohistochemical detection of ER and PR, as well as HER2 is routinely carried out to allow the selection of patients who will benefit from antihormonal or HER2-targeted therapy. As the basal group of breast cancers has substantial overlap with the triple-negative phenotype, the identification of ERBB2- or basal-like cancers poses no problem. However, separating hormone-receptor positive tumors into low-risk and high-risk (luminal $\mathrm{A}$ and luminal B) cancers or patients with lower or greater benefit from cytotoxic chemotherapy requires additional information. Including proliferation data (such as Ki-67 immunostains) into the decision process has been a promising approach, as luminal A tumors typically contain smaller growth fractions as compared with luminal B tumors. ${ }^{7}$ However, there are important limitations to this approach: Different studies have used cutoffs of between 5 and $20 \%$ Ki-67-positive nuclei for the differentiation of low-risk and high-risk cancers, ${ }^{7-10}$ but the vast number of cases within this range, as well as tumor heterogeneity and (importantly) an overall poor interobserver agreement hampered this approach. ${ }^{11}$ Furthermore, as proliferation rates typically decrease following cytotoxic chemotherapy, it is impossible to assess comparable proliferation data once any adjuvant or neo-adjuvant treatment has been undertaken. ${ }^{12}$

In the present study, we have chosen a different approach to overcome these limitations. By evaluating the expression of a several well-characterized elements of the ER pathway, including steroid receptors, cofactors and -repressors, as well as interacting growth factor receptors, transcription factors, and oncogenes, we sought to determine individual factors or patterns differentiating lowrisk from high-risk luminal tumors in a consecutive series of postmenopausal patients that were selected as a five-hospital sub-cohort of a larger, epidemiologic series. To facilitate the identification of the other breast cancer subtypes and to compare any observed risk stratification with the established proliferation cutoffs, we included TP53, HER2, and high-molecular cytokeratins (CK5/14), as well as Ki-67-stains.

\section{Materials and methods}

From an initial series of 1739 postmenopausal patients diagnosed with breast cancer between 2002 and $2005,{ }^{13,14}$ we included a subcollective of 443 patients who had been treated in eight of the participating hospitals from the Rhine-NeckarKarlsruhe study region based on the availability of paraffin-embedded tumor tissue. Patients who had received neo-adjuvant cytotoxic chemotherapy were excluded. Clinical and pathological data are summarized in Table 1. Mean follow-up was 57 months (range, 0-89 months). Average patient age at diagnosis was 63 years (range, 50-75 years).

Paraffin-embedded tumor tissues were collected from the archives of the Institute of Pathology of the Heidelberg University with approval of the local Ethics committee. We constructed a set of tissue microarrays containing triplicates of $0.6 \mathrm{~mm}$ cores from different areas of tumor and normal tissue as described previously. ${ }^{15}$

Immunostains were performed using the avidinbiotin complex method andaminoethylcarbazol (AEC) as chromogen (Chemmate Immunoperoxidase/AEC Kit, Dako, Hamburg, Germany). Following heat-induced epitope retrieval in citrate buffer (pH6), antibodies against ERalpha (clone 1D5, 1:50, Dako), PR (clone PGR636, 1:50, Dako), HER2 (polyclonal, 1:500, Dako), BCL2 (clone 124, 1:100, Dako), TP53 (clone DO7, 1:100, Dako), CK5/14 (clones XM26 + LL002, 1:100, Novocastra/Leica, Nussloch, Germany) or Ki-67 (MIB1, 1:100, Dianova, Hamburg, Germany) were incubated at room temperature for 25 min. For ERbeta (clone 14C8, Genetex, Irvine, CA, USA), SRC-1 (clone 1135/H4, Santa Cruz, Heidelberg, Germany), GATA3 (clone HG3-31, Santa Cruz), FOXA1 (clone 2D7, Abnova, Heidelberg, Germany), P300 (polyclonal, Santa Cruz) and IGF1R (polyclonal, Santa Cruz), pretreatment was performed using $1 \mathrm{~mm}$ EDTA, pH9 followed by antibody incubation at $4{ }^{\circ} \mathrm{C}$ over night. EGFR staining (clone 31G5, 1:30, Zytomed, Berlin, Germany) was performed after proteolytic pretreatment with pronase E $(0.1 \% \mathrm{w} / \mathrm{v}$ in $\mathrm{PBS}, 6 \mathrm{~min}$ at $37^{\circ} \mathrm{C}$ ).

For Ki-67 and TP53 stains, the percentage of positively stained tumor cell nuclei was recorded for each core, for HER2 and EGFR, the three-tiered Dako-Scores were used. BCL2 was evaluated semiquantitatively $(0-3+)$, for $\mathrm{CK} 5 / 14$ and IGF1R, the percentage of positively stained cells was recorded while for the rest of the markers, percentage of positive cells, as well as intensity of staining (0-3) was recorded and subsequently used to calculate immunoreactive scores. ${ }^{16}$ ER and PR stains were considered positive in cases with $>1 \%$ positively stained nuclei as proposed by the ASCO guidelines. ${ }^{17}$ In addition, we also calculated 
Table 1 Clinical and pathological characterization of the 443 consecutive breast carcinomas and the four observed clusters

\begin{tabular}{|c|c|c|c|c|c|}
\hline & Total & Luminal A & Luminal $B$ & $E R B B 2$ & $T N B C$ \\
\hline & 443 & $147(33.2 \%)$ & $224(50.6 \%)$ & $39(8.8 \%)$ & $33(7.4 \%)$ \\
\hline Average patient age & 63 & 62 & 63 & 61 & $60(P=0.038)$ \\
\hline \multicolumn{6}{|l|}{ pT-stage } \\
\hline pTis & 19 & $5(26.3 \%)$ & $5(26.3 \%)$ & $6(31.6 \%)$ & $3(15.8 \%)$ \\
\hline pT1 & 227 & $88(38.8 \%)$ & $116(51.1 \%)$ & $14(6.2 \%)$ & $9(4 \%)$ \\
\hline pT2 & 160 & $46(28.8 \%)$ & $82(51.3 \%)$ & $14(8.8 \%)$ & $18(11.3 \%)$ \\
\hline pT3 & 20 & $5(25 \%)$ & $11(55 \%)$ & $2(10 \%)$ & $2(10 \%)$ \\
\hline pT4 & 16 & $3(18.8 \%)$ & $9(56.3 \%)$ & $3(18.8 \%)$ & $1(6.3 \%)$ \\
\hline pTx & 1 & & & & \\
\hline \multicolumn{6}{|l|}{ pN-stage } \\
\hline pNo & 231 & $81(35.1 \%)$ & $118(51.1 \%)$ & $16(6.9 \%)$ & $16(6.9 \%)$ \\
\hline pN1 & 87 & $27(31 \%)$ & $46(52.9 \%)$ & $6(6.9 \%)$ & $8(9.2 \%)$ \\
\hline $\mathrm{pN} 2$ & 24 & $7(29.2 \%)$ & $12(50 \%)$ & $3(12.5 \%)$ & $2(9.2 \%)$ \\
\hline pN3 & 30 & $5(16.7 \%)$ & $20(66.7 \%)$ & $3(10 \%)$ & $2(6.7 \%)$ \\
\hline $\mathrm{pNX}$ & 71 & & & & \\
\hline \multicolumn{6}{|l|}{ Lymphangiosis } \\
\hline L0 & 285 & $103(36.1 \%)$ & $149(52.3 \%)$ & $19(6.7 \%)$ & $14(4.9 \%)$ \\
\hline L1 & 120 & $34(28.3 \%)$ & $60(50 \%)$ & $13(10.8 \%)$ & $13(10.8 \%)$ \\
\hline (No information) & 38 & & & & \\
\hline \multicolumn{6}{|l|}{ Grading } \\
\hline G1 & 60 & $30(50 \%)$ & $29(48.3 \%)$ & 0 & $1(1.7 \%)$ \\
\hline $\mathrm{G} 2$ & 251 & $92(36.7 \%)$ & $141(56.2 \%)$ & $9(3.6 \%)$ & $9(3.6 \%)$ \\
\hline G3 & 129 & $24(18.6 \%)$ & $53(41.1 \%)$ & $30(23.3 \%)$ & $22(17.5 \%)$ \\
\hline (No information) & 3 & & & & \\
\hline \multicolumn{6}{|l|}{ Histologic type } \\
\hline Invasive ductal & 307 & $96(31.1 \%)$ & $149(48.5 \%)$ & $32(10.4 \%)$ & $30(9.8 \%)$ \\
\hline Invasive lobular & 87 & $27(31.0 \%)$ & $51(58.6 \%)$ & $2(2.3 \%)$ & 0 \\
\hline Tubular & 20 & $8(40 \%)$ & $12(60 \%)$ & 0 & 0 \\
\hline Ductal in situ & 19 & $7(36.8 \%)$ & $5(26.3 \%)$ & $5(26.3 \%)$ & $2(10.5 \%)$ \\
\hline Mucinous & 4 & $2(50 \%)$ & $2(50 \%)$ & 0 & 0 \\
\hline Micropapillary & 2 & $2(100 \%)$ & 0 & 0 & 0 \\
\hline Medullary & 2 & 0 & $1(50 \%)$ & 0 & $1(50 \%)$ \\
\hline Other & 2 & $1(50 \%)$ & $1(50 \%)$ & 0 & 0 \\
\hline
\end{tabular}

immunoreactive scores for ER and PR to allow for the testing of different cutoff-values incorporating staining intensity.

Designation of biological subtypes based on the results of ER, PR, Ki-67, and HER2-stains was performed according to the St Gallen consensus applying a proliferation cutoff of $>14 \% .{ }^{18}$ To calculate IHC4-scores, the original formula by Cuzick et $a l^{18}$ was used: $94.7 \times\left(-0.100 \quad \mathrm{ER}_{10}-0.079\right.$ $\mathrm{PR}_{10}+0.586$ HER2 + 0.240 ln $\left.(1+10 \times \mathrm{Ki}-67)\right)$. Immunoreactive scores for ER and PR were rescaled to a range of $0-10$ by using a multiplier of 0.8333 . HER2-scores ranging from 0 to 3 were entered into the formula without normalization. On the basis of the distribution of mean and s.d., Ki-67 percentages were adjusted by using a multiplier of 0.4 as described by Cuzick et al ${ }^{18}$ and Barton et al. ${ }^{19}$

Unsupervised hierarchical clustering was performed using the $\mathrm{R}$ software package ( $\mathrm{R}$ 2.13.2, http://www.cran.org). After normalization of the data to values between 0 and 1 (with 1 representing the maximum score for each marker), the heatmap2 function was used to plot heatmap and trees and to extract the exact position of each tumor sample within the cluster. For further statistical analysis, Fisher's exact test was used for parametric data and Wilcoxon's rank test for non-parametric data. Testing was performed two-sided, $P$-values $<0.05$ were considered significant. Uni- and multivariate survival analysis were performed using log-rank tests and the Cox proportional hazards model.

\section{Results}

Hierarchical cluster analysis using the immunohistochemically detected expression data of ERalpha, ERbeta, PR, HER2, BCL2, TP53, EGFR, IGF1R, SRC-1, GATA3, FOXA1, AIB1, P300, CK5/14, and Ki-67 reproduced the four established, biologically distinct breast cancer subtypes (Figure 1). The two larger groups contained estrogen receptor positive (luminal) tumors that were separated by progesterone receptor and BCL2 expression. HER2positive tumors were clustered together regardless of ER-coexpression and most triple-negative tumors 


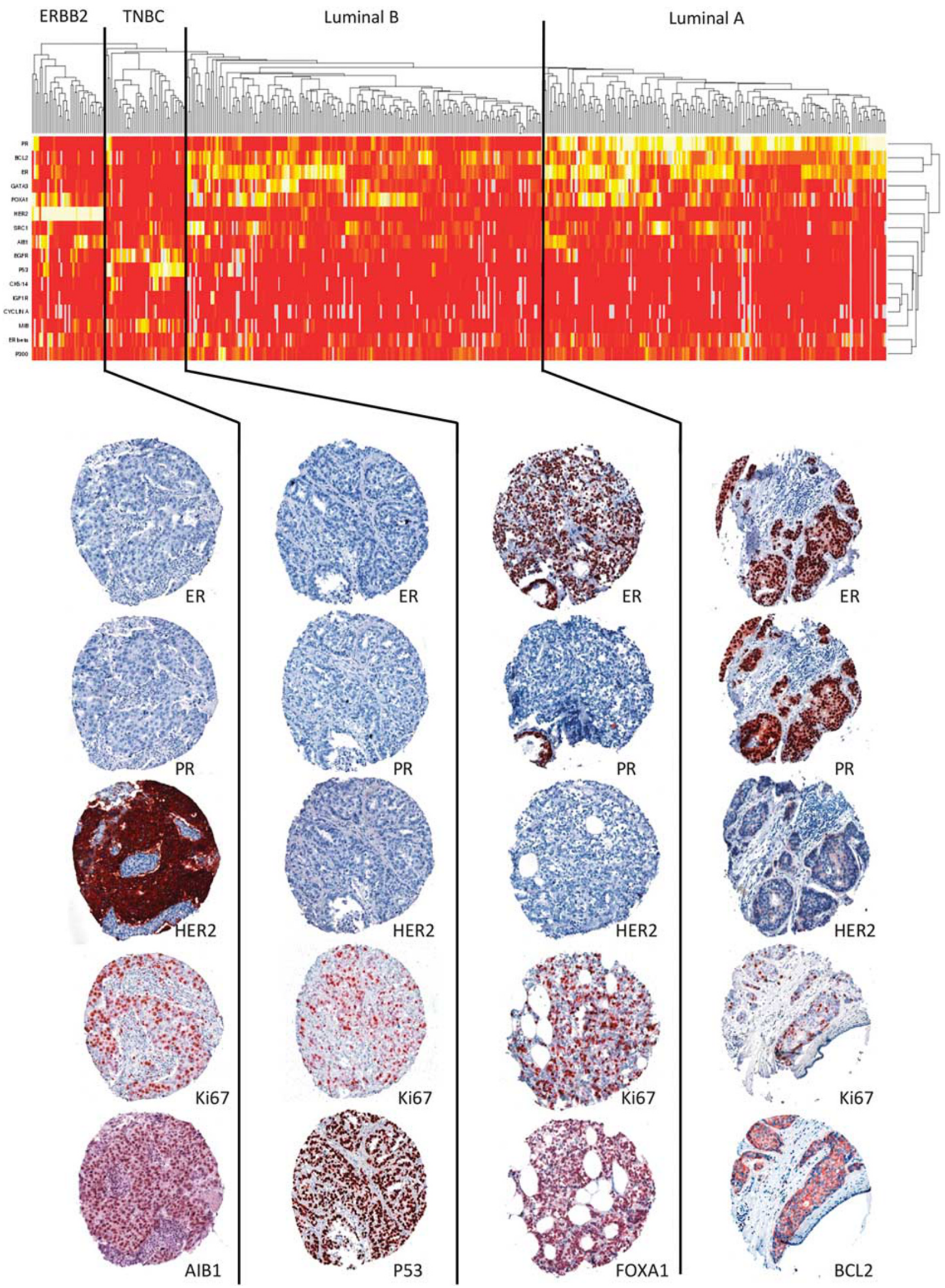

Figure 1 Top: unsupervised clustering of 443 breast carcinomas using immunohistochemically detected estrogen receptor signalingassociated proteins, proliferation, growth factor receptors, as well as HER2, CK5/14, EGFR and TP53. Bottom: exemplary immunostains for each of the four main clusters. 
were grouped within the fourth group. The detailed staining results and clinico-pathological characteristics of the four groups are summarized in Tables 1 and 2 .

Survival analysis demonstrated significant differences in progression-free survival between the four clusters $(P=0.0006$ for all four groups, Figure 2a). Importantly, stratification of luminal tumors into luminal A (good prognosis) and the more-aggressive luminal $B$ subtype was highly significant $(P=0.002)$. In comparison, the immunohistochemical classification of biological subtypes according to the St Gallen consensus showed significant differences between luminal, basal and HER2 clusters $(P=0.0004$, Figure $2 \mathrm{~b})$ but failed to stratify luminal tumors into two distinct (luminal $\mathrm{A}$ and $\mathrm{B}$ ) subgroups with prognostic differences $(P=0.658)$. At present, most immunohistochemistry-based classification schemes use proliferation (with varying cutoff-values) to separate luminal B from luminal A tumors. The St Gallen consensus has proposed a Ki-67-cutoff of $>14 \%^{18}$ but different studies have used values between 5 and $20 \%$ (7-10). However, in our series, Ki-67-cutoffs within this range failed to produce prognostically distinct subgroups (Figure 3). To further investigate a possible role of interobserver variation, we compared the interpretation of Ki-67-stains in estrogen- or progesterone receptor-positive tumors between both a graduate student (LB) and an experienced breast pathologist (SA). Although there was a bias towards lower Ki67-scores for one of the investigators (LB), the overall interpretation was strongly correlated (rho $=0.778, P<0.0001$, Spearman's rank correlation, Figure 4a). As Ki-67-stains had already been performed on full tissue sections at the time of primary diagnosis in 225 of the cases, we were able to compare these values with the data obtained from the tissue microarrays. Although still significantly associated, lower correlation coefficients were observed ( $\mathrm{rho}=0.610$ and $\mathrm{rho}=0.483$, respectively, $P<0.0001$, Spearman's rank correlation, Figures $4 \mathrm{~b}$ and c) with a substantial number of cases being grouped inconsistently when applying the Ki-67cutoff of $>14 \%$ to the different Ki-67 data sets. Accordingly, survival analysis demonstrated significant differences between luminal $\mathrm{A}$ and B tumors only when the St Gallen criteria were applied to Ki-67 scores from full tissue sections while the evaluation of three different tissue microarray cores failed to reproduce this classification (Figure $4 \mathrm{~d}-\mathrm{f}$ ). To test whether a different Ki-67-cutoff might be a

Table 2 Staining results within the four clusters

\begin{tabular}{|c|c|c|c|c|c|}
\hline & Luminal $A$ & Luminal B & ERBB2 & $T N B C$ & Interpretation \\
\hline PR & $\begin{array}{l}147 / 147(100 \%) \\
147 / 147(100 \%) \\
141 / 147(95.9 \%)\end{array}$ & $\begin{array}{c}123 / 223(55.2 \%) \\
97 / 223(43.5 \%) \\
39 / 223(17.5 \%)\end{array}$ & $\begin{array}{l}9 / 39(23.1 \%) \\
6 / 39(15.4 \%) \\
5 / 39(12.8 \%)\end{array}$ & $\begin{array}{c}5 / 33(15.2 \%) \\
4 / 33(12.2 \%) \\
2 / 33(6.1 \%)\end{array}$ & $\begin{array}{c}>1 \% \text { Pos. nuclei } \\
\text { Score } \geq 2 \\
\text { Score } \geq 6\end{array}$ \\
\hline BCL2 & 135/136 (99.3\%) & $178 / 210(84.8 \%)$ & $10 / 35(28.6 \%)$ & $10 / 30(33.3 \%)$ & Any staining \\
\hline ERalpha & $\begin{array}{c}130 / 147(88.4 \%) \\
117 / 147(79.6 \%) \\
81 / 147(55.1 \%)\end{array}$ & $\begin{array}{c}186 / 224(83 \%) \\
166 / 224(74.1 \%) \\
92 / 224(41.1 \%)\end{array}$ & $\begin{array}{c}16 / 39(41 \%) \\
12 / 39(30.8 \%) \\
5 / 39(12.8 \%)\end{array}$ & $\begin{array}{c}7 / 33(21.2 \%) \\
3 / 33(9.1 \%) \\
1 / 33(3 \%)\end{array}$ & $\begin{array}{c}>1 \% \text { Pos. nuclei } \\
\text { Score } \geq 2 \\
\text { Score } \geq 6\end{array}$ \\
\hline GATA3 & $69 / 132(52.3 \%)$ & $111 / 204(54.4 \%)$ & $10 / 37(27 \%)$ & $2 / 32(6.3 \%)$ & Score $\geq 2$ \\
\hline FOXA1 & $62 / 123(50.4 \%)$ & 129/195 (66.2\%) & $25 / 36(69.4 \%)$ & $3 / 32(9.4 \%)$ & Score $\geq 2$ \\
\hline HER2 & $0 / 147(0 \%)$ & $1 / 224(0.4 \%)$ & $38 / 39(97.4 \%)$ & $0 / 33(0 \%)$ & Score $\geq 2$ \\
\hline Ki-67 (tissue microarray) & 10.2 & 11.3 & 22.8 & 43 & Mean (\%) \\
\hline Ki-67 (full section) & 10.2 & 14.7 & 45.5 & 60.8 & Mean (\%) \\
\hline EGFR & $1 / 133(0.8 \%)$ & $5 / 206(2.4 \%)$ & $3 / 36(8.3 \%)$ & $14 / 31(45.2 \%)$ & Score $\geq 2$ \\
\hline P53 & $1 / 142(0.7 \%)$ & $9 / 211(4.3 \%)$ & $5 / 38(13.2 \%)$ & $17 / 32(53.1 \%)$ & Score $\geq 2$ \\
\hline IGF1R & $8 / 134(6 \%)$ & $20 / 206(9.7 \%)$ & $2 / 37(5.4 \%)$ & $2 / 31(6.5 \%)$ & Any membr. staining \\
\hline CK5/14 & $2 / 135(1.5 \%)$ & $4 / 203(2 \%)$ & $2 / 37(5.4 \%)$ & $12 / 32(37.5 \%)$ & $\geq 10 \%$ Pos. cells \\
\hline CYCLIN A & $7 / 125(5.6 \%)$ & $14 / 189(7.4 \%)$ & $7 / 32(21.9 \%)$ & 10/31 (32.3\%) & $\geq 10 \%$ Pos. nuclei \\
\hline AIB1 & $7 / 145(4.8 \%)$ & $17 / 211(8.1 \%)$ & $16 / 38(42.1 \%)$ & $2 / 33(6.1 \%)$ & Score $\geq 2$ \\
\hline ERBETA & $114 / 127(89.8 \%)$ & 159/192 (82.8\%) & $32 / 35(91.4 \%)$ & $21 / 30(70 \%)$ & Any staining \\
\hline P300 & $69 / 144(47.9 \%)$ & $107 / 218(49.1 \%)$ & $24 / 38(63.2 \%)$ & $18 / 32(56.3 \%)$ & Score $\geq 2$ \\
\hline
\end{tabular}



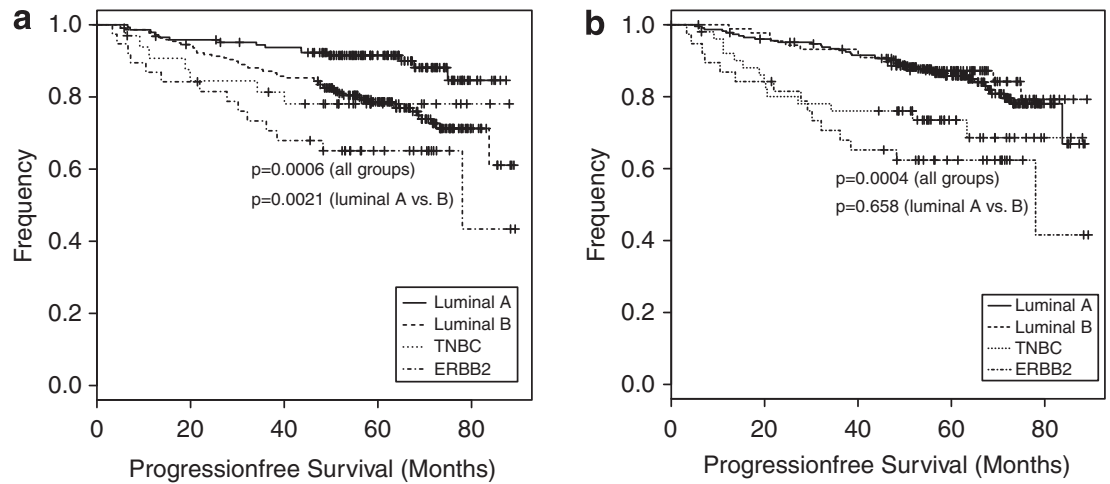

Figure 2 Progression-free survival in the four breast cancer subgroups as detected by cluster analysis (a) or defined according to the St Gallen consensus (b). Note that luminal A and B groups demonstrate significantly different survival only in the detected clusters (A, $P=0.0021)$, but not when applying the St Gallen consensus $(B, P=0.658)$.
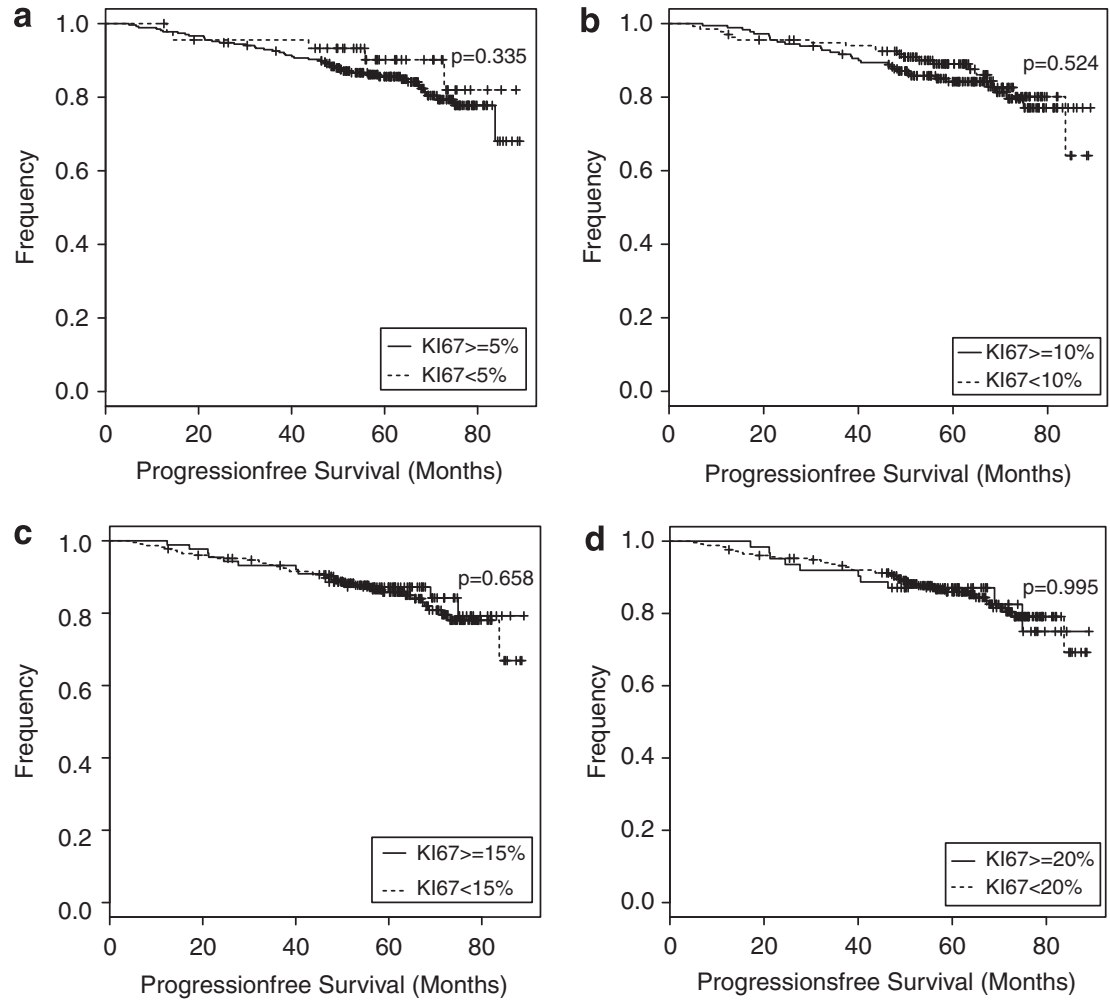

Figure 3 Progression-free survival of luminal breast cancers stratified by different Ki-67-cutoffs (Ki-67-stains on tissue microarrays), $(\mathbf{a}, \mathbf{b}): \geq 5 \%$ and $\geq 10 \%$ as used by Millar et al, (8) (c) $\geq 15 \%$ as suggested by the St Gallen Consensus, ${ }^{18}$ and (d) $\geq 20 \%$ as determined by Jacquemier et al. ${ }^{7}$

better prognosticator, receiver operator characteristic-curves using the proliferation data from full tissue sections identified the optimum cutoff-value for the prognosis of a poorer patient outcome was not $14 \%$, but $20 \%$ Ki-67-positive nuclei in our series (Figure 5a).

As progesterone receptor expression was the most important stratifyer between luminal A and B tumors in our cluster analysis, we used receiver operator characteristic-curves to test for the best cutoff-value to predict a poorer prognosis in luminal breast tumors (Figure 5b). The calculated value of 2 corresponds to $10 \%$ positively stained nuclei with a moderate staining intensity. Multivariate analysis using a Cox proportional hazards model confirmed the independant relevance of PR-expression (Table 3). In fact, looking at luminal tumors only, lack of PR-expression was the second most important predictor of an adverse patient outcome (odds ratio 2.43, 95\% confidence interval (CI) $1.40-4.22 ; P=0.0015$, see Table 3 ) following lymph node metastasis. This predictive value remained significant when looking at early, stage 1 (luminal tumors, pT1 or 2, no lymph node metastases: odds ratio $=2.68,95 \%$ CI $1.03-6.95, P=0.043$ ) tumors only. Proliferation, grading, and tumor extent (pT category) did not differ between $\mathrm{PR}^{+}$and

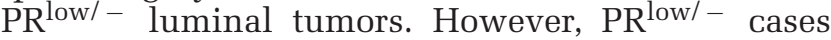



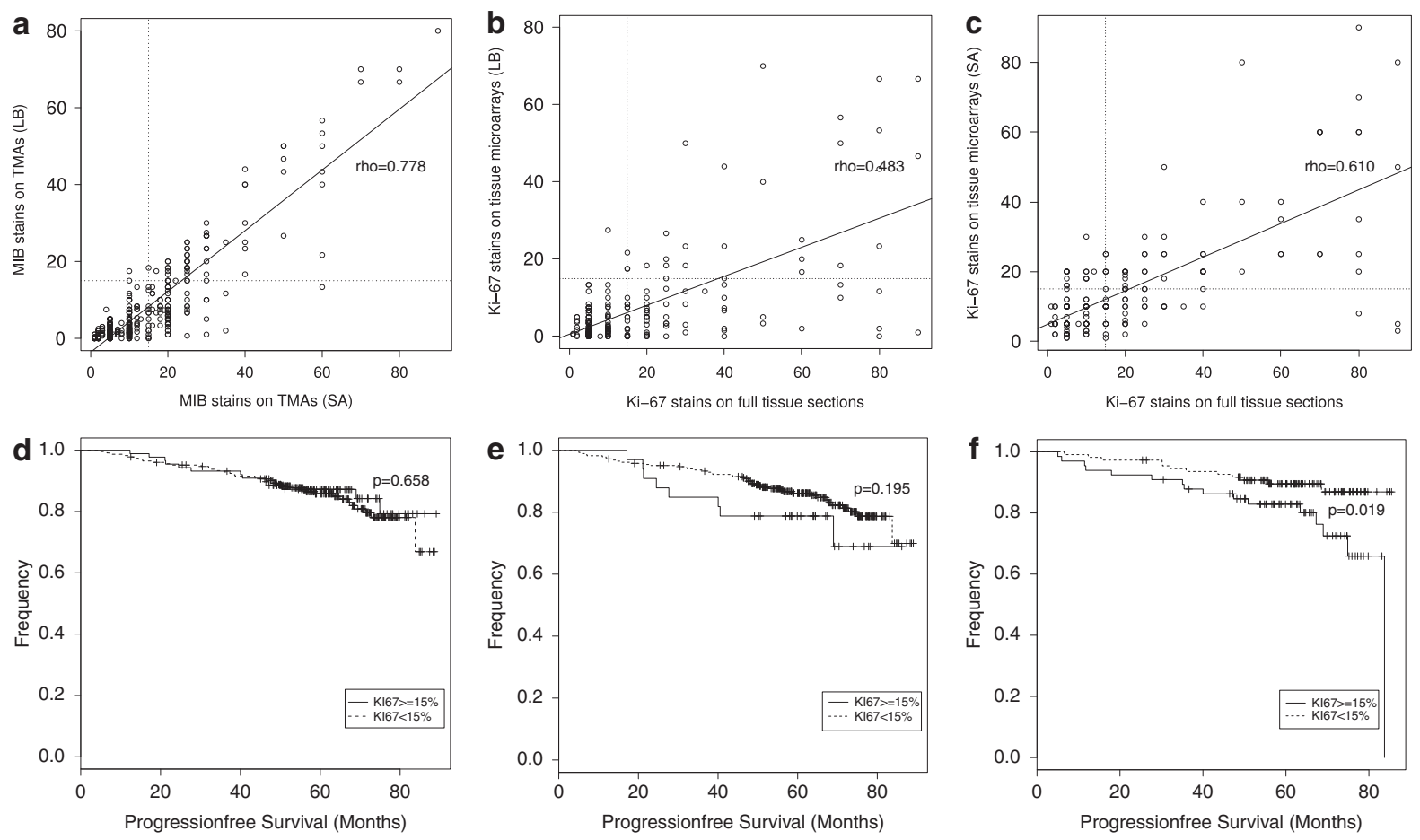

Figure 4 Good overall correlation (a) of Ki-67 stainining interpretation by two investigators on the same set of tissue microarrays. However, poorer correlation coefficients are reached comparing tissue microarrays with prospectively evaluated Ki-67-stains on full tissue sections (b, c). Survival analysis demonstrates a significant risk stratification only when using Ki-67 scores from full tissue sections, but not from tissue microarray cores $(\mathbf{d}-\mathbf{f})$.

a

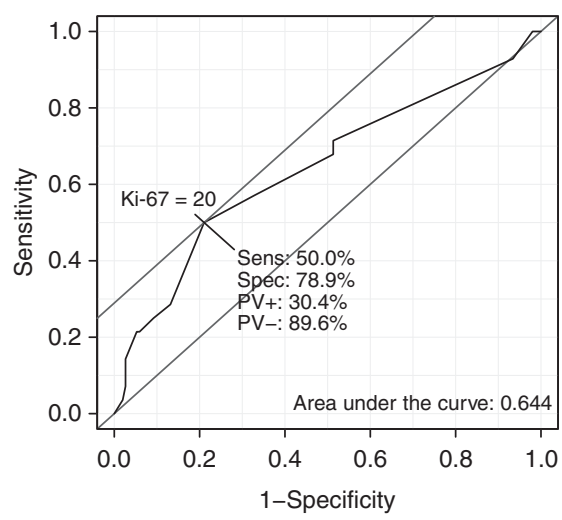

b

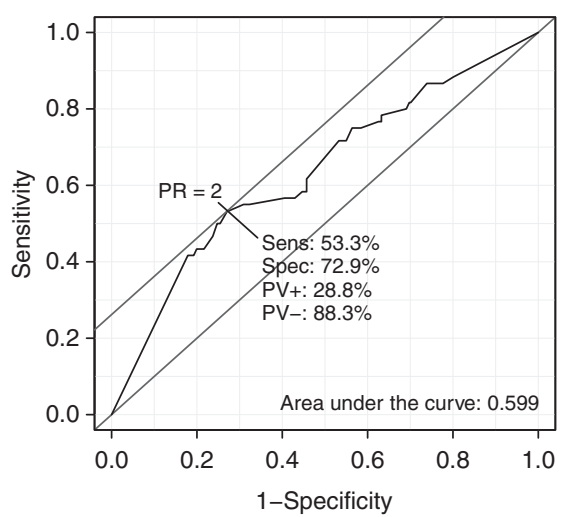

Figure 5 Receiver operator characteristic curve analysis of (a) Ki-67 (full tissue sections) and (b) PR stains (immunoreactive scores). Optimum cutoff-values are indicated within the graphs.

Table 3 Multivariate analysis using a cox proportional hazards model on all tumors and luminal tumors only

\begin{tabular}{lccc}
\hline & All tumors & & Luminal tumors \\
\hline & Odds ratio (95\% CI) & $\mathrm{P}$ & Odds ratio (95\% CI) \\
pT & $2.37(1.35-4.15)$ & 0.0026 & $1.81(0.89-3.68)$ \\
pN & $1.90(1.12-3.22)$ & 0.0169 & $2.77(1.53-5.04)$ \\
Grading (G3) & $2.04(1.24-3.36)$ & 0.0049 & $2.24(1.27-3.93)$ \\
PR weak/negative & $2.48(1.49-4.12)$ & 0.0005 & $2.43(1.40-4.22)$ \\
HER2 positive & $1.29(0.64-2.60)$ & 0.48 & 0.0008 \\
ER positive & $1.28(0.73-2.26)$ & 0.39 & \\
& & & \\
\hline
\end{tabular}

Abbreviation: CI, confidence interval.

Weak or negative PR status is defined as immunoreactive score $<2$ or $<10 \%$ positively stained nuclei. 

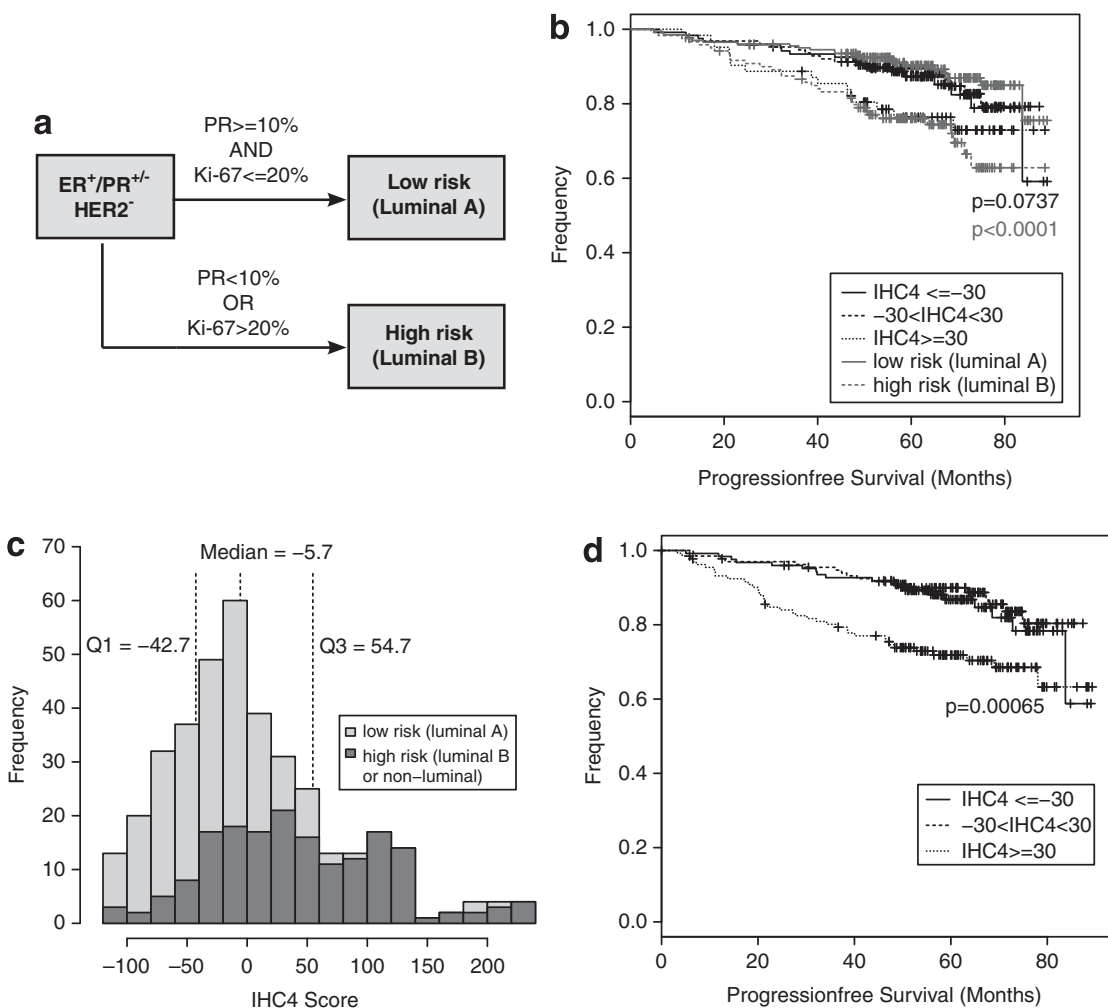

Figure 6 Risk classification and survival analysis in luminal breast tumors incorporating PR staining and Ki-67 using cutoff-values determined by receiver operator characteristic-curve analysis (see text). (a) Tumors with PR-/low phenotype or increased proliferation ( $>20 \%$ Ki-67-positive nuclei) are classified as high risk/luminal B while $\mathrm{PR}^{\text {high }}$-tumors with Ki-67 $\leq 20 \%$ are considered low risk/ luminal A. Progression-free survival of patients with luminal tumors is shown in panel (b). Red lines show low-/high-risk classification based on Ki-67 and PR-expression, black lines demonstrate risk stratification according to the IHC4-score. (c) Distribution of IHC4-scores in low- (light gray) and high-risk (dark gray) breast carcinomas. The median score, 1st and 3rd quartiles of all included breast carcinomas are indicated within the graph (d). Prognostic relevance of the IHC4 classifier in unselected postmenopausal breast carcinoma patients. Cutoff-values were $<-30$ for low risk and $>30$ for high risk (see Cuzik et al ${ }^{18}$ ).

were associated with a higher number of positive lymph nodes (pN-stages 2 and $3, P=0.022$, Fisher's exact test).

To test, whether the determination of estrogen- or progesterone receptor expression on tissue microarray cores correlates with results obtained from full tissue sections under routine conditions (available in 439 cases), spearman correlations were calculated and demonstrated a high rho value for PR (rho $=0.759, P<0.0001)$. For ER, a somewhat lower rho was observed (0.633, $P<0.0001)$.

In order to test the combination of Ki-67 and PR for risk classification of luminal breast cancers under conditions approximating core biopsies, tumors were grouped into low risk and high risk based on Ki-67 and PR stains from the tissue microarray cores. For this classification, we used the cutoff-values determined by receiver operator characteristic-curve analysis (tumors with low or no PR-expression $(<10 \% /$ immunoreactive score $<2)$ or with high Ki-67 scores (>20\%)) were classified high risk while the rest of the cases was considered low risk (Figure 6a). The progression-free survival of the low-risk group (PR $\geq 2 / \geq 10 \%$ and Ki-67 $\leq 20 \%)$ was substantially better than in the high-risk group on univariate (odds ratio $=2.80,95 \%$ CI
1.64-4.79, $P<0.0001$ ) and multivariate (odds ratio $=2.53,95 \%$ CI $1.42-4.53, P=0.0017)$ analysis. The resulting Kaplan-Meier plot is demonstrated in Figure 6b, red lines. In comparison, the IHC4-score also provided a significant risk stratification in our series when applied to all cases (Figures 6c and d). However, looking at luminal tumors only, the IHC4score showed only a trend towards poorer patient survival that failed to reach significancy (high-risk group vs intermediate- and low-risk tumors, $P=0.0737$, Figure 6b).

\section{Discussion}

The molecular classification of breast carcinomas into four major subtypes (luminal A, luminal B, ERBB2, and basal-like) has not only separated tumors into different groups with characteristic molecular alterations, but also with distinct biological behavior. ${ }^{20}$ The luminal A subtype is characterized by high expression of ER, as well as a number of ER-targets and a low overall proliferation while the (higher-risk) luminal B subtype expresses not only estrogen receptors but a number of cell-cycle associated RNAs, as well as receptor tyrosine kinase-signaling associated genes. ${ }^{21,22}$ 
On the basis of these findings, endocrine therapy is believed to be more effective in the luminal A group while the luminal B subtype seem to represent cancers, in which endocrine therapy may fail and additional cytotoxic chemotherapy may have a higher benefit. ${ }^{23}$

As attempts for a prospective tumor classification using expression profiling have been difficult, ${ }^{6}$ the St Gallen international expert panel has proposed surrogate definitions using routinely available, conventional immunohistochemical stains for ER, PR, HER2, and Ki-67. Still, the distinction of ER + / HER2 - luminal breast cancers into (low-risk) luminal $\mathrm{A}$ and (higher-risk) luminal $\mathrm{B}$ subtypes based on Ki-67-stains has turned out to be difficult due to interobserver variability and the substantial heterogeneity within the tumor tissue. ${ }^{24}$

Luminal A and luminal B substantially differ regarding the mechanisms mediating cell proliferation and survival. While in luminal A tumors conventional nuclear transcriptional activity of the estrogen receptor is believed to confer important growth stimuli, luminal B tumors are characterized by activation of non-canonical ER signaling, as well as receptor cross-talk with tyrosine kinases leading to an activation of PI3K- and/or MAPK-signaling. ${ }^{25}$ To evaluate whether the immunohistochemical detection of parts of the ER signaling network may be used to distinguish the two biologically and prognostically distinct types of luminal tumors, we performed unsupervised hierarchical clustering of a series of postmenopausal breast carcinomas using a set of proteins involved in estrogen receptor signaling or associated with resistance to endocrine therapy in combination with established breast cancer-related proteins. Similar to previous studies that applied hierarchical clustering to cDNA data, we identified two distinct luminal (estrogen receptor positive) subtypes, one group of HER2associated tumors and a group of triple-negative tumors. Surprisingly, neither proliferation nor the expression of one or more of the ER-cofactors or resistance-associated factors, but PR-expression was identified as the most important stratifyer between the luminal subtypes in our series. In fact, not only the four identified clusters were shown to be significantly associated with patient outcome, PR-expression alone could be used to stratify $\mathrm{ER}^{+}$tumors into a low-risk and a high-risk group. The outcome of $\mathrm{PR}^{\text {low/ }}$ - luminal tumors remained significantly poorer in multivariate analysis, as well as in subgroup analyses of localized and stage 1 carcinomas.

The hypothesis that PR-expression predicts response to endocrine therapy has been proposed by Horwitz et al ${ }^{25,26}$ more than 30 years ago. On the cellular level, PR-negative luminal tumors are characterized by increased growth factor signaling, ${ }^{27,28}$ as well as an activation of non-canonical ER-signaling ${ }^{29,30}$ resulting in an increased activation of the PI3K and MAPK pathways. Two large patient series analyzed by Bardou et $a l^{31}$ demonstrated a substantially poorer patient outcome of $\mathrm{ER}^{+} / \mathrm{PR}^{-}$ tumors following anti-hormonal treatment using tamoxifen. Although the use of aromatase inhibitors instead of selective estrogen receptor modulators has improved the outcome of $\mathrm{ER}^{+} / \mathrm{PR}^{-}$ patients in the ATAC trial, ${ }^{32}$ results from the Breast International Group demonstrate not only a highly significant association of PR-expression with disease-free survival in ER-expressing tumors but also a higher efficacy of the aromatase inhibitor letrozole over tamoxifen in $\mathrm{ER}^{+} / \mathrm{PR}^{+}$-tumors, which failed to reach significancy for $\mathrm{ER}^{+} / \mathrm{PR}^{-}$tumors. ${ }^{33}$

Our data demonstrate that assessing PR-expression allows for the prospective stratification of luminal tumors into a low-risk and a high-risk group. Although the identification of $\mathrm{PR}$ as the single most important stratifyer between both luminal groups in our series came as a surprise, a closer look at ER biology may explain this finding as the $P R$ gene is a classical target of canonical ER signaling following nuclear ER activation in the presence of a functioning network of cofactors. ${ }^{34,35}$ In the context of either selective estrogen receptor modulators blocking canonical ER action or inactivation of some of the cofactors, the ER interacts with growth factor receptors leading to an activation of MAPK or PI3K signaling, but no transcription of ER-targets such as the $P R .^{22}$ Interestingly, PR is an important part not only of the PAM50 gene signature, a set of 50 genes that can be used to determine the intrinsic subtype of a given tumor sample, ${ }^{36}$ but also of the 21-gene recurrence score (Oncotype DX, Genomic Health Inc., Redwood City, CA, USA). ${ }^{37}$ In the latter, PR-expression in fact has a $50 \%$ higher influence on the ER-subscore than the ER itself while in the PAM50 signature, high PR levels are characteristic of luminal A, but not of luminal B tumors.

However, while our data show that loss of PRexpression is an important predictor of poorer patient outcome in $\mathrm{ER}^{+}$breast carcinomas, occasionally aggressive tumors with molecular or immunohistochemical evidence of activated alternative signaling mechanisms may be PR positive and thereby missed when using PR-expression alone to classify luminal tumors into low- and high-risk cases. This is especially true for HER2-amplified breast cancers, some of which still express ER and PR (so-called 'triple-positive' tumors). Although these 'triple-positive' cases may be diagnosed as luminal B tumors according to the St Gallen consensus, ${ }^{18}$ our unsupervised cluster analysis grouped them next to the other HER2-amplified/ overexpressing cases. In the clinical setting, it may be best to clearly relate the HER2-positivity in these cases just to the therapeutic implications. However, as a number of other growth factor receptors, including EGFR, IGF1R, and IR may also be activated in luminal B breast carcinomas ${ }^{38,39}$ resulting in increased proliferation, we tested a 
risk classification, in which loss of PR-expression $(<10 \%$ positive nuclei/immunoreactive score $<2)$ or high tumor proliferation ( $>20 \%$ Ki-67-positive nuclei) were used to define luminal tumors as high risk. Although the cutoff-values for PR and Ki-67 were based on receiver operator characteristic-curve analysis with progression-free survival as stratifier, the same cutoffs for both PR and Ki-67 had also been used by previous studies to discriminate prognostically divergent groups of tumors. ${ }^{8,40,41}$

In our series, this classification was superior to the conventional classification according to the St Gallen criteria, as well as the immunohistochemical IHC4-score in predicting patient survival. The IHC4-score has previously been shown to provide similar prognostic information as the 21-gene recurrence score in breast cancer patients. ${ }^{19}$ In combination with clinico-pathological data (grade, tumor size, extent of lymph node metastases and anti-hormonal treatment) it can be used for the risk stratification of early breast carcinomas in a prospective way. ${ }^{20}$ We can confirm the prognostic value of the IHC4-score when looking at unselected breast tumors. However, when applied to HER2negative, luminal tumors, the simplified low-risk and high-risk (or luminal A/luminal B) classification based on PR and $\mathrm{Ki}-67$-stains provides a better stratification, which can be attributed to the influence of HER2 and ER on the score. In addition, Ki-67 immunostains have been shown to show the greatest intratumoral variability in tumors with moderate proliferation rates (around $10-20 \%$ positive nuclei), ${ }^{24}$ so that in our hands, a combination of PR and Ki-67 renders the classification of breast tumors into luminal A (low risk) and luminal B (high risk) more reliable.

In conclusion, our data demonstrate, that assessment of PR-expression using conventional immunostains allows for the distinction of high-risk and low-risk ER ${ }^{+}$(luminal) breast cancers reflecting the biological activity of canonical ER-signaling in the tumor cells. In addition, combining PR-expression with a higher Ki-67-cutoff (20\%) may overcome the limitations of the current St Gallen consensus to more reliably stratify ER-positive tumors into luminal A (low risk) and luminal B (high risk).

\section{Acknowledgements}

The authors wish to thank Sandra Hahn, Tina Philipp, Ursula Eilber, and Christina Krieg for excellent technical assistance. This work was supported by grants from the Dietmar Hopp Stiftung and the Deutsche Krebshilfe (project numbers 108253/108419/108418).

\section{Disclosure/conflict of interest}

The authors declare no conflict of interest.

\section{References}

1 Lin SX, Chen J, Mazumdar M, et al. Molecular therapy of breast cancer. Progress and future directions. Nat Rev Endocrinol 2010;6:485-493.

2 Chumsri S, Howes T, Bao T, et al. Aromatase, aromatase inhibitors and breast cancer. J Steroid Biochem Mol Biol 2011;125:13-22.

3 Perou CM, Sørlie T, Eisen MB, et al. Molecular portraits of human breast tumours. Nature 2000;406: 747-752.

4 Sørlie T, Perou CM, Tibshirani R, et al. Gene expression patterns of breast carcinomas distinguish tumor subclasses with clinical implications. Proc Natl Acad Sci USA 2001;98:10869-10874.

5 Geyer FC, Rodrigues DN, Weigelt B, et al. Breast cancer molecular profiling with single sample predictors: a retrospective analysis. Lancet Oncol 2010;11:339-349.

6 Cheang MC, Chia SK, Voduc D, et al. Ki67 index, HER2 status, and prognosis of patients with luminal B breast cancer. J Natl Cancer Inst 2009;101:736-750.

7 Jacquemir J, Charafe-Jauffret E, Monville F, et al. Association of GATA3, P53, Ki67 status and vascular peritumoral invasion are strongly prognostic in luminal breast cancer. Breast Cancer Res 2009;11:R23.

8 Millar EK, Graham PH, McNeil CM, et al. Prediction of outcome of early ER + breast cancer is improved using a biomarker panel, which includes Ki-67 and p53. Br J Cancer 2011;105:272-280.

9 Fasching PA, Heusinger K, Haeberle L, et al. Ki67, chemotherapy response, and prognosis in breast cancer patients receiving neoadjuvant treatment. BMC Cancer 2011;11:486.

10 Dowsett M, Nielsen TO, A'Hern R, et al. Assessment of Ki67 in breast cancer: recommendations from the International Ki67 in Breast Cancer working group. J Natl Cancer Inst 2011;103:1656-1664.

11 Assersohn L, Salter J, Powles TJ, et al. Studies of the potential utility of Ki67 as a predictive molecular marker of clinical response in primary breast cancer. Breast Cancer Res Treat 2003;82:113-123.

12 Buck K, Vrieling A, Zaineddin AK, et al. Serum enterolactone and prognosis of postmenopausal breast cancer. J Clin Oncol 2011;29:3730-3738.

13 Vrieling A, Buck K, Heinz J, et al. Pre-diagnostic alcohol consumption and postmenopausal breast cancer survival: a prospective patient cohort study. Breast Cancer Res Treat 2012;136:195-207.

14 Aulmann S, Bentz M, Sinn HP. C-myc oncogene amplification in ductal carcinoma in situ of the breast. Breast Cancer Res Treat 2002;74:25-31.

15 Remmele W, Stegner HE. Vorschlag zur einheitlichen definition eines Immunreaktiven Score (IRS) fur den immunhistochemischen Ostrogenrezeptor-Nachweis (ER-ICA) im Mammakarzinomgewebe. Pathologe 1987; 8:138-140.

16 Hammond ME, Hayes DF, Dowsett M, et al. American Society of Clinical Oncology/College of American Pathologists guideline recommendations for immunohistochemical testing of estrogen and progesterone receptors in breast cancer. J Clin Oncol 2010;28: 2784-2795.

17 Goldhirsch A, Wood WC, Coates AS, et al. Strategies for subtypes-dealing with the diversity of breast cancer: highlights of the St Gallen International Expert Consensus on the Primary Therapy of Early Breast Cancer 2011. Ann Oncol 2011;22:1736-1747. 
18 Cuzick J, Dowsett M, Pineda S, et al. Prognostic value of a combined estrogen receptor, progesterone receptor, Ki-67, and human epidermal growth factor receptor 2 immunohistochemical score and comparison with the genomic health recurrence score in early breast cancer. J Clin Oncol 2011;29:4273-4278.

19 Barton S, Zabaglo L, A'Hern R, et al. Assessment of the contribution of the IHC4 $+\mathrm{C}$ score to decision making in clinical practice in early breast cancer. Br J Cancer 2012;106:1760-1765.

20 Jönsson G, Staaf J, Vallon-Christersson J, et al. Genomic subtypes of breast cancer identified by array-comparative genomic hybridization display distinct molecular and clinical characteristics. Breast Cancer Res 2010;12:R42.

21 Cui X, Schiff R, Arpino G, et al. Biology of progesterone receptor loss in breast cancer and its implications for endocrine therapy. J Clin Oncol 2006;23:7721-7735.

22 Kao KJ, Chang KM, Hsu HC, et al. Correlation of microarray-based breast cancer molecular subtypes and clinical outcomes: implications for treatment optimization. BMC Cancer 2011;18:143.

23 Varga Z, Diebold J, Dommann-Scherrer C, et al. How reliable is Ki-67 immunohistochemistry in grade 2 breast carcinomas? A QA study of the Swiss working group of breast- and gynecopathologists. PloS One 2012;7:e37379.

24 Badve S, Nakshatri H. Oestrogen-receptor-positive breast cancer: towards bridging histopathological and molecular classifications. J Clin Pathol 2009;62:6-12.

25 Horwitz KB, McGuire WL. Predicting response to endocrine therapy in human breast cancer. A hypothesis. Science 1975;189:726-727.

26 Horwitz KB, McGuire WL. Estrogen control of progesterone receptor in human breast cancer. Correlation with nuclear processing of estrogen receptor. J Biol Chem 1978;253:2223-2228.

27 Cui X, Zhang $\mathrm{P}$, Deng W, et al. Insulin-like growth factor-I inhibits progesterone receptor expression in breast cancer cells via the phosphatidylinositol 3kinase/Akt/mammalian target of rapamycin pathway: progesterone receptor as a potential indicator of growth factor activity in breast cancer. Mol Endocrinol 2003;17:575-588.

28 Arpino G, Weiss H, Lee AV, et al. Estrogen receptorpositive, progesterone receptor-negative breast cancer: association with growth factor receptor expression and tamoxifen resistance. J Natl Cancer Inst 2005;97:1254-1261.

29 Campbell RA, Bhat-Nakshatri P, Patel NM, et al. Phosphatidylinositol 3-kinase/AKT-mediated activation of estrogen receptor alpha: a new model for antiestrogen resistance. J Biol Chem 2001;276:9817-9824.

30 Faridi J, Wang L, Endemann G, et al. Expression of constitutively active Akt-3 in MCF-7 breast cancer cells reverses the estrogen and tamoxifen responsivity of these cells in vivo. Clin Cancer Res 2003;9: 2933-2939.

31 Bardou VJ, Arpino G, Elledge RM, et al. Progesterone receptor status significantly improves outcome prediction over estrogen receptor status alone for adjuvant endocrine therapy in two large breast cancer databases. J Clin Oncol 2003;21:1973-1979.

32 Howell A, Cuzick J, Baum M, et al. Results of the ATAC (Arimidex, Tamoxifen, Alone or in Combination) trial after completion of 5 years' adjuvant treatment for breast cancer. Lancet 2005;365:60-62.

33 Viale G, Regan MM, Maiorano E, et al. Prognostic and predictive value of centrally reviewed expression of estrogen and progesterone receptors in a randomized trial comparing letrozole and tamoxifen adjuvant therapy for postmenopausal early breast cancer: BIG 1-98. J Clin Oncol 2007;25:3846-3852.

34 Bradshaw MS, Tsai SY, Leng XH, et al. Studies on the mechanism of functional cooperativity between progesterone and estrogen receptors. J Biol Chem 1991;266:16684-16690.

35 Petz LN, Ziegler YS, Schultz JR, et al. Differential regulation of the human progesterone receptor gene through an estrogen response element half site and Sp1 sites. J Steroid Biochem Mol Biol 2004;88: 113-122.

36 Parker JS, Mullins M, Cheang MC, et al. Supervised risk predictor of breast cancer based on intrinsic subtypes. J Clin Oncol 2009;27:1160-1167.

37 Paik S, Shak S, Tang G, et al. A multigene assay to predict recurrence of tamoxifen-treated, node-negative breast cancer. N Engl J Med 2004;351:2817-2826.

38 Potter AS, Casa AJ, Lee AV. Forkhead box A1 (FOXA1) is a key mediator of insulin-like growth factor I (IGF-I) activity. J Cell Biochem 2012;113:110-121.

39 Creighton CJ. The molecular profile of luminal B breast cancer. Biologics 2012;6:289-297.

40 Ogawa Y, Moriya T, Kato Y, et al. Immunohistochemical assessment for estrogen receptor and progesterone receptor status in breast cancer: analysis for a cut.off point as the predictor for endocrine therapy. Breast Cancer 2004;11:267-275.

41 Yamashita H, Yando Y, Nishio M, et al. Immunohistochemical evaluation of hormone receptor status for predicting response to endocrine therapy in metastatic breast cancer. Breast Cancer 2006;13:74-83. 